\title{
Comparative Analysis of a Hierarchical Bayesian Method for Quantitative Trait Loci Analysis for the Arabidopsis Thaliana
}

\author{
Caroline Pearson ${ }^{1}$, Susan J. Simmons ${ }^{1}$, Karl Ricanek Jr. ${ }^{2}$, and Edward L. Boone ${ }^{3}$ \\ ${ }^{1}$ University of North Carolina Wilmington, Department of Mathematics and Statistics, \\ ${ }^{2}$ University of North Carolina Wilmington, Department of Computer Science, \\ 601 South College Road, Wilmington, North Carolina, 28403, USA \\ ${ }^{3}$ Virginia Commonwealth University, \\ Department of Statistical Sciences and Operations Research, \\ Richmond, Virginia, 23284, USA \\ caroline.pearson310@gmail.com, \{simmonssj, ricanekk\}@uncw. edu, \\ elboone@vcu.edu
}

\begin{abstract}
This work performs an analysis on two, quite different, techniques for Quantitative Trait Loci (QTL) Analysis. Interval Mapping (IM) as described by Karl Broman is compared to a Hierarchical Bayesian Model (HBM) technique that reduces the problem of QTL analysis down to one of model selection. Simulations were generated for the flowering plant of the Arabidopsis thaliana for evaluation of the techniques. It is shown that the HBM technique was much more successful at determining the appropriate loci/markers and corresponding chromosomes than the IM technique given a single loci. It was further elucidated through simulation runs that the HBM was robust against two loci/markers, whereas IM completely failed. The contribution of this work is in the comparison and analysis of the IM method to that of the HBM; hence, demonstrating through simulations that the HBM technique is superior to that of the IM for the Arabidopsis simulated data.
\end{abstract}

Keywords: Quantitative Trait Loci (QTL) Analysis, Arabidopsis, Interval Mapping, Hierarchical Bayesian Model.

\section{Introduction}

The commencement of the genomic era has witnessed an increased interest in identifying locations on a genome responsible for a quantitative trait, referred to as quantitative trait loci (QTL). Mapping a quantitative trait to a location on a genome is made possible through a genetic map, which illustrates the relative distance between known markers or genes on an organism's genome. Alfred Henry Sturtevant constructed the first genetic map in 1913, and the first analysis relating genes to quantitative traits was done in 1923 by Sax [1]. Since these initial works and a few others, not much else has been done in the area of QTLs until the 1980's. 
The last two decades has seen an explosion of algorithms proposed for the identification of QTLs. A few of the more notable initial methods include Lander and Botstein [2]; Jensen [3]; Zeng [4]; Wright and Mowers [5]; Kearsey and Hyne [6]; $\mathrm{Wu}$ and Li [7]; and, Sen and Churchill [8]. In the last decade a technique known as interval mapping [9], in which pseudo-markers were placed in the interval between two known markers to evaluate the possibility of a QTL in the interval, has dominated the research landscape. Variations on the Interval Mapping (IM) algorithm such as Composite Interval Mapping [4, 10] and Multiple Interval Mapping [11] have also gained much attention in the 1990's. Yet, still other influential methods have been proposed to analyze QTLs; for example, generalized estimating equations [2], partial least squares [12], and Bayesian approaches [13, 14, 15, 16, 17]. Each method has its own advantages and weaknesses; however, all of these approaches assume only one observation per genotype.

A single trait is usually determined by many genes; as a result, many QTLs are usually associated with a single trait. The number of QTLs associated with each phenotypic trait tells us the genetic makeup and the variation of this trait. For instance, a small effect can be determined if there are many QTLs correlated with a single trait and a large effect can be determined if there are only a few QTLs correlated with a single trait. The information gleamed from the QTL can help us better understand the chemical structure of these traits and better understand the evolution of these traits over a period of time. Furthermore, QTLs can ultimately enable the alteration of the chemical structure of these traits. One potential benefit of understanding the QTLs for plants is the ability to alter the chemical structure of a plant to make it more tolerant of ultraviolet (UV) radiation, which may help agriculturalists deal with the depleting ozone layer; this layer filters much of the UV radiation before it can enter the atmosphere and ultimately the terra firma.

As mentioned above, most QTL methods have been developed mainly for human and animal genotypes. Since all humans and animals have unique genetic composition, each observation per genotype (or line) consists of a single observation value. However, plants are often cloned and their genetic makeup reproduced, generating multiple observations per genotype (or line). The clones within each line consist of the same marker information on their genetic makeup; therefore the question must be asked, Are the methods for human and animal genotypes appropriate for plant genotypes?

Current state-of-the-art algorithms for QTL detection are limited to the use of a single value for the quantitative trait per genotype; therefore, plant biologists will take the mean or median value of the quantitative trait within each line to perform their analysis. Although, plants have the unique capability of being cloned, important information, like variability within a line, may be lost due to the suppression of data into a single value for methods such as interval mapping. The hierarchical Bayesian model (HBM) proposed in [18] and further elucidated for QTL analysis in [19] over comes the limitation of a single value for the quantitative trait. The following sections will detail the development of the hierarchical Bayesian model used for this work (section 2 Methods), the simulations that were generated to evaluate the IM against HBM (section 3 Simulations), the results (section 4) and conclusions drawn (section 5). 


\subsection{Arabidopsis Thaliana}

The Arabidopsis thaliana, which is illustrated in figure 1, is an angiosperm, a dicot from the mustard (Brassicaceae) family. This little plant has become to plant biology what Drosophila melanogaster and Caenorhabditis elegans are to animal biology. Although this plant has no commercial viability, it has proved to be an ideal organism for the study of plant development.

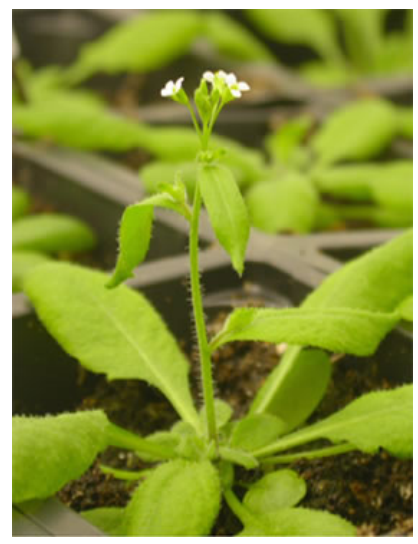

Fig. 1. Arabidopsis thaliana member of the mustard (Brassicaceae) family, which is shown here a grow tray

The main attractions of this plant as a model organism for cellular and molecular biology of flowering plants are, short germination to seed maturation (6 weeks); seed production is prolific and the plant is easily cultivated; large number of mutant lines and genomic resources are available; extensive genetic and physical maps of all 5 chromosomes are readily available; one of the smallest genomes in the plant kingdom with little junk DNA; mutations can be easily generated; and it is self-pollinated so recessive mutations become quickly homozygous.

\section{Methods}

Hierarchical models have proven to be invaluable in many instances (e.g. Boone et al. [18] and Simmons et al. [20]). In the case of plant QTL experiments, hierarchical models make the most sense and are flexible enough to adequately model the data. The response, $y_{i j}$ is the numerical value of the quantitative trait for $i=1, \ldots, L$ and $j=$ $1, \ldots, n_{i}$ where $L$ is the number of lines and $n_{i}$ represents the number of replicates within each line. Each $y_{i j}$ is assumed to be linearly dependent on the genetic composition of the plant, in other words,

$$
y_{i j}=\beta_{0}+\beta_{1} x_{i 1}+\beta_{2} x_{i 2}+\ldots \beta_{M} x_{i M}
$$


where $x_{i j}= \begin{cases}1 & \text { if marker from parent A } \\ 0 & \text { if marker from parent B }\end{cases}$

and $M$ is the number of markers. Most models for QTL experiments involve similar forms of a linear association between the quantitative trait and the markers. However, most QTL models assume that there is only one observation per genotype, or per line, and that the variance is the same within each line $[2,20]$. The hierarchical model does not make this assumption of homogeneity of variance and is able to incorporate the replicate information within each line.

The hierarchical model assumes that each $y_{i j}$ is normally distributed with a mean of $\theta_{i}$ and a variance of $\sigma_{i}^{2}$. The next level of the hierarchy assumes that the $\theta_{i}$ 's are normally distributed with a mean of $\beta_{0}+\beta_{1} x_{i 1}+\beta_{2} x_{i 2}+\ldots \beta_{M} x_{i M}$ and a variance of $\tau^{2}$. This model allows different variances within each line $\left(\sigma_{i}^{2}\right)$ and a variance between the lines $\left(\tau^{2}\right)$. The Bayesian paradigm is extremely flexible and easy to incorporate hierarchical structures evident in these experiments. The following prior distributions will be assumed

$$
\begin{gathered}
\beta_{m} \sim \mathrm{N}(0,100) \\
\tau^{2} \sim \text { Inverse- } \chi^{2}(2) \\
\sigma_{i}^{2} \sim \text { Inverse- } \chi^{2}(2) .
\end{gathered}
$$

The Inverse- $\chi^{2}(2)$ is a natural choice for the variances, since it has an infinite variance (Boone et al. [22]). The prior distribution for the $\beta$ s s assume that no markers have an effect on the quantitative trait, and forces the data to dictate which markers are most important with respect to the quantitative trait.

Combining this information into a hierarchical model creates a full joint posterior distribution of the form

$$
\begin{gathered}
p\left(\theta, \beta, \sigma^{2}, \tau^{2} \mid y\right) \propto\left(\tau^{\tau_{0}+2+L} \prod_{i}\left(\sigma_{i}^{n_{i}+\sigma_{0 i}+2}\right)\right)^{-1} . \\
\exp \left[-\sum_{i} \frac{1}{2 \sigma_{i}^{2}}-\frac{1}{2 \tau^{2}}-\frac{1}{200} \beta^{\prime} \beta-\frac{1}{2 \tau^{2}}(\theta-X \beta)^{\prime}(\theta-X \beta)-\sum_{i} \sum_{j} \frac{1}{2 \sigma_{i}^{2}}\left(y_{i j}-\theta_{i}\right)^{2}\right] .
\end{gathered}
$$

The Gibbs Sampler, a Markov Chain Monte Carlo technique, can generate samples from the full joint posterior distribution in (5) by using the following conditional posterior distributions

$$
\begin{gathered}
p\left(\tau^{2} \mid \theta, \beta, \sigma^{2}, y\right) \sim \operatorname{Inv}-\operatorname{Gamma}\left(\frac{L+\tau_{0}^{2}}{2}, \frac{(\theta-X \beta)^{\prime}(\theta-X \beta)+1}{2}\right) \\
p\left(\sigma_{i}^{2} \mid \theta, \beta, \tau^{2}, y\right) \sim \operatorname{Inv}-\operatorname{Gamma}\left(\frac{n_{i}+\sigma_{0}^{2}}{2}, \frac{\sum_{j}\left(y_{i j}-\theta_{i}\right)^{2}+1}{2}\right)
\end{gathered}
$$




$$
\begin{gathered}
p\left(\beta \mid \tau^{2}, \theta, \sigma^{2}, y\right) \sim N\left(\left(\frac{I}{100}+\frac{X^{\prime} X}{\tau^{2}}\right) \frac{X^{\prime} \theta}{\tau^{2}},\left(\frac{I}{100}+\frac{X^{\prime} X}{\tau^{2}}\right)^{-1}\right) \\
p\left(\theta \mid \tau^{2}, \beta, \sigma^{2}, y\right) \sim N\left(\frac{\frac{X_{i} \beta}{\tau^{2}}+\frac{n_{i} \bar{y}_{i}}{\sigma_{i}^{2}}}{\frac{1}{\tau^{2}}+\frac{n_{i}}{\sigma_{i}^{2}}}, \frac{1}{\frac{1}{\tau^{2}}+\frac{n_{i}}{\sigma_{i}^{2}}}\right) .
\end{gathered}
$$

This information can be used to find posterior probabilities and ultimately determine which markers are important in controlling the quantitative trait. For example, the posterior probability of interest is the probability of model $\eta_{i}$ given the data

$$
P\left(\eta_{i} \mid D\right)=\frac{P\left(D \mid \eta_{i}\right) P\left(\eta_{i}\right)}{\sum_{j=1}^{|\mathrm{H}|} P\left(D \mid \eta_{i}\right) P\left(\eta_{i}\right)},
$$

where $P\left(\eta_{i}\right)$ is the prior probability of model $\eta_{i}$ and $|\mathrm{H}|$ is the set of all possible models. Assuming no prior information is known about the markers, equal probability is assigned to each $\eta_{i}$. The quantity $P\left(D \mid \eta_{i}\right)$ in equation (10) is calculated by

$$
P\left(D \mid \eta_{i}\right)=\int P\left(D \mid \omega_{i}, \eta_{i}\right) P\left(\omega_{i} \mid \eta_{i}\right) d \omega_{i},
$$

where $\omega_{i}$ is the vector of unknown parameters under model $\eta_{i}$. This integration is computationally intensive, but can be estimated by

$$
\int P\left(D \mid \omega_{i}, \eta_{i}\right) P\left(\omega_{i} \mid \eta_{i}\right) d \omega_{i} \approx \frac{1}{t} \sum_{j=1}^{t} P\left(D \mid \omega_{i}^{(j)}, \eta_{i}\right),
$$

where $\omega_{i}^{(j)}$ are samples from the full posterior distribution under model $\eta_{i}$. Since there are many unknown parameters in this model, a large number of samples from the posterior are recommended. In this research, $t$ was chosen to be 100,000 with a burn-in period of 2,000. The posterior probability of the model given the data can be used to find the activation probability of a marker or region, $\mathrm{P}\left(\beta_{j} \neq 0 \mid D\right)$. The activation probability is defined as,

$$
P\left(\beta_{j} \neq 0 \mid D\right)=\sum_{|\mathrm{H}|} P\left(\beta_{j} \neq 0 \mid \eta_{i}, D\right) P\left(\eta_{i} \mid D\right) \cdot
$$

However, to calculate the activation probability for each marker means that $2^{M}$ models need to be fit. This can become computationally intensive since most genetic maps have more than 100 markers ( $M$ is generally larger than 100). Therefore, a search strategy is needed. We define a conditional search strategy that continues to break the genome into smaller and smaller regions and retains only those regions of importance.

The search strategy first breaks the genome into chromosome regions. In this instance, there are $2^{K}$ number of models that need to be evaluated where $K$ is the number of chromosomes. The activation probability for each region is evaluated by

$$
P\left(\kappa_{j} \neq 0 \mid D\right)=\sum_{|\mathrm{H}|} P\left(\kappa_{j} \neq 0 \mid \eta_{i}, D\right) P\left(\eta_{i} \mid D\right) \cdot
$$


Regions with posterior probability larger than 0.5 are regarded as potential QTLs and retained in the model. Once all potential regions are identified, those regions retained are divided in half. For example, in a hypothetical example with 7 chromosomes, the search algorithm would first find which chromosomes make a significant contribution to the QTL by searching through all $2^{7}=128$ possible models and calculating the activation probability for each chromosome. For this example, the following activation probabilities were obtained $\mathrm{C}_{1}=0.01, \mathrm{C}_{2}=0.03, \mathrm{C}_{3}=0.67, \mathrm{C}_{4}=$ $0.33, \mathrm{C}_{5}=0.90, \mathrm{C}_{6}=0.21$ and $\mathrm{C}_{7}=0.84$. Chromosomes 3,5 and 7 have activation probabilities higher than 0.5 and are kept for further analysis. Dividing these chromosomes in half, there are now six regions to explore (i.e. $2^{6}=64$ models). These regions are defined as $\mathrm{C}_{31}, \mathrm{C}_{32}, \mathrm{C}_{51}, \mathrm{C}_{52}, \mathrm{C}_{71}$ and $\mathrm{C}_{72}$. The algorithm is rerun and activation probabilities for each of these six regions are calculated. Only those regions with activation probability higher than 0.5 are retained and then divided in half. This algorithm is repeated until the activation probabilities are calculated on individual markers.

\section{Simulations}

The dataset that was utilized for the $\mathrm{X}$ matrix (165 lines x 38 markers) is from a real marker structure, the Bay-0 x Shahdara population created by Oliver Loudet and Sylvian Chaillou [23] which is depicted below in Figure 1.

A genetic map is a map based on the frequencies of recombination between markers during crossover of homologous chromosomes. The greater the frequency of recombination (segregation) between two genetic markers, the farther apart they are assumed to be. Conversely, the higher the frequency of association between the markers, the smaller the physical distance between them [24]. Distance on a genetic map is measured in centiMorgans $(\mathrm{cM})$ which is a relative distance between two markers.

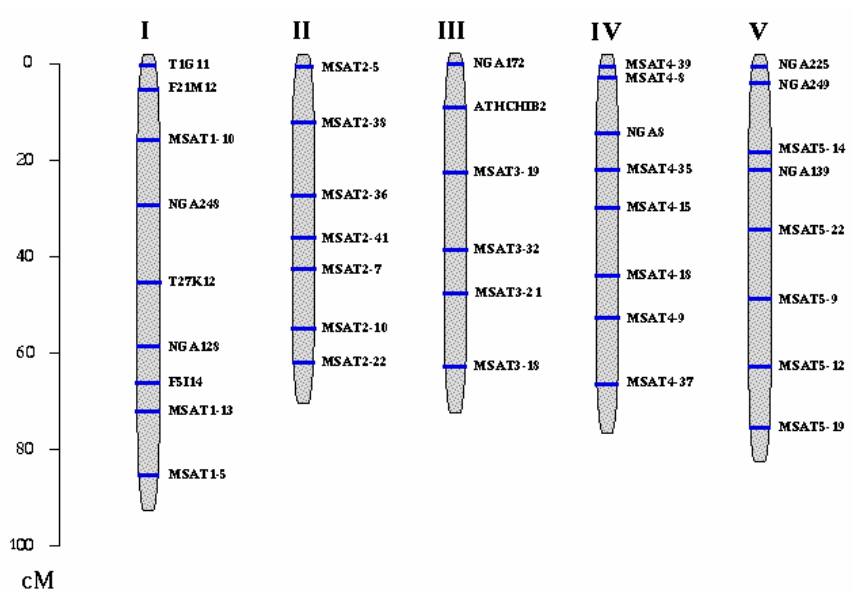

Fig. 2. The genetic map of the Arabidopsis Thaliana Bay-O by Shahdara 
The genetic map of the Bay-0 $\mathrm{x}$ Shahdara population is made up of five chromosomes consisting of approximately 6 to 9 markers each. Marker values $\left(x_{i}\right)$ were set to $x_{i}=0,0.5$ or 1 . The marker value $x_{i}=0$ came from parent $\boldsymbol{A}$ and the marker $x_{i}=1$ came from parent $\boldsymbol{B}$, whereas, the marker value $x_{i}=0.5$ is a missing or unknown value. Ten response values $\left(y_{i j}\right)$ were simulated per line around approximately a 26 unit mean $(\mu)$ which depended on the QTL location and the actual genotypic trait. Bimodal standard deviations $\left(\sigma_{1}\right.$ and $\left.\sigma_{2}\right)$ were created around the mean value $(\mu)$ for the observations in each line. The response values $\left(y_{i j}\right)$ for the one QTL simulation were drawn from a random normal distribution. The marker values of the QTL were simulated as follows,

$$
y_{i j}=\mu+2 * a_{i} * x_{i}+\varepsilon_{i j},
$$

where, $\mu=$ the underlying true mean and $a_{i}=$ QTL affect, $x_{i}=0,0.5$ or 1 and $\varepsilon_{i j}$ is random error noise. The random error noise $\varepsilon_{i j}$ has standard deviation $\sigma_{1}$ or $\sigma_{2}$ depending on a random draw from a Bernoulli with probability of success 0.5 .

In the case of two QTLs, the following equation was used to simulate the QTL value,

$$
y_{i j}=\mu+a_{1} * x_{1 i}+a_{2} * x_{2 i}+\varepsilon_{i j}
$$

where, $\mu=$ the underlying true mean and $\mathrm{a}_{1}, \mathrm{a}_{2}=$ QTL affects and $\varepsilon_{i j}$ is random error noise, as defined previously.

One or two QTLs were arbitrarily placed on or around random markers with two different effects as shown in Table 1 and Table 2. The Bay-0 x Shahdara X-matrix and the simulated response values were run through the two methods introduced in section one to see which one performed better given the different variances created.

Table 1. Simulations for one QTL that were used for this work. The chromosome were the marker of choice is located (ground truth) given the effect and number of QTL's.

\begin{tabular}{|c|l|l|}
\hline \multirow{2}{*}{ Effects } & \multicolumn{1}{|c|}{ Standard Deviations } \\
\cline { 2 - 3 } & \multicolumn{1}{|c|}{$\boldsymbol{\sigma}_{\mathbf{1}}=\mathbf{2 . 0}, \boldsymbol{\sigma}_{\mathbf{2}} \mathbf{= 4 . 5}$} & \multicolumn{1}{c|}{$\boldsymbol{\sigma}_{\mathbf{1}}=\mathbf{4 . 2}, \boldsymbol{\sigma}_{\mathbf{2}}=\mathbf{9 . 1}$} \\
\hline \multirow{2}{*}{$\mathbf{a}=\mathbf{2}$} & $\begin{array}{l}\text { Chrom 3 } \\
\text { M 18 }\end{array}$ & $\begin{array}{l}\text { Chrom 5 } \\
\text { M 36 }\end{array}$ \\
\hline \multirow{2}{*}{$\mathbf{a}=\mathbf{1 2}$} & $\begin{array}{l}\text { Chrom 3 } \\
\text { M 15 }\end{array}$ & $\begin{array}{l}\text { Chrom 2 } \\
\text { M 12 }\end{array}$ \\
\hline
\end{tabular}

The two methods being evaluated in this sensitivity study have different criteria associated with their respective selection mechanism for QTL markers. The Interval Mapping method uses the Logarithm of the Odds (LOD), shown in equation (17), such that any loci with a score greater than a threshold value is said to be a potential QTL. From [20] it has been stated that a LOD score of 11 or greater are deemed significant; hence a marker selection can be determined.

$$
\text { LOD }=-2 \ln \left[\frac{\max (\text { likelihood assuming no QTLs })}{\max (\text { likelihood assuming QTL at location })}\right] \text {. }
$$


Table 2. Simulations for two QTLs that were used for this work. The chromosome were the marker of choice is located (ground truth) given the effect and number of QTL's.

\begin{tabular}{|c|l|l|}
\hline \multirow{2}{*}{ Effects } & \multicolumn{2}{|c|}{ Standard Deviations } \\
\cline { 2 - 3 } & \multicolumn{1}{|c|}{$\boldsymbol{\sigma}_{\mathbf{1}}=\mathbf{1 . 5}, \boldsymbol{\sigma}_{\mathbf{2}} \mathbf{= 2 . 5}$} & \multicolumn{1}{|c|}{$\boldsymbol{\sigma}_{\mathbf{1}} \mathbf{= 2 . 0}, \boldsymbol{\sigma}_{\mathbf{2}} \mathbf{= 4 . 5}$} \\
\hline \multirow{2}{*}{$\mathbf{a}_{\mathbf{1}}=\mathbf{1}$} & Chrom 1 & Chrom 2 \\
$\mathbf{a}_{\mathbf{2}}=\mathbf{2}$ & M 5 & M 11 \\
& Chrom 4 & Chrom 5 \\
& M 24 & M 36 \\
\hline & Chrom 2 & Chrom 1 \\
$\mathbf{a}_{\mathbf{1}}=\mathbf{1}$ & M 14 & M 8 \\
$\mathbf{a}_{\mathbf{2}}=\mathbf{2}$ & Chrom 3 & Chrom 3 \\
& M 21 & M 18 \\
\hline & Chrom 1 & Chrom 2 \\
$\mathbf{a}_{\mathbf{1}}=\mathbf{2}$ & M 5 & M 11 \\
$\mathbf{a}_{\mathbf{2}}=\mathbf{1 2}$ & Chrom 4 & Chrom 5 \\
& M 24 & M 36 \\
\hline
\end{tabular}

HBM as stated above in the Methods section (section 2) uses a conditional activation probability to determine loci of interest. It is further stated that a conditional activation probabilities of 0.50 or greater is deemed significant. Hence, probability scores of 0.50 are used to determine the loci of markers.

\section{Results}

The locations of the simulated QTLs illustrated in Tables 1 and 2 were arbitrarily chosen for this study. Small effect sizes (1 and 2) and large effect sizes were chosen to study the sensitivity of the methods to the size of the effect. In a similar fashion, larger and smaller variances were also chosen to study the influence of different variations.

Table 3 clearly indicates that the HBM is able to detect the simulated QTLs. In three instances in Table 3 the HBM also detects adjoining markers, which is not uncommon in QTL analysis. Markers that are close together on a genetic map tend to be highly correlated, so often more than one marker will be detected in a QTL analysis. Table 3 also indicates that the IM algorithm can detect the approximate location of the QTL; however, the IM algorithm tends to choose the general region of the QTL by selecting the marker immediately after the simulated QTL.

Table 4 illustrates the two QTL case where the HBM again is able to detect the correct locations of the QTLs and occasionally picks up adjacent markers. The IM algorithm is only able to detect the larges effect size of 12 in the study. Effect sizes of 1 and 2 are not detected in the IM algorithm. 
Table 3. One QTL Summary Table. ( ${ }^{1}$ Final conditional activation probability. ${ }^{2}$ LOD score.)

\begin{tabular}{|c|c|c|c|c|}
\hline \multirow{3}{*}{ Effects } & \multicolumn{4}{|c|}{ Standard Deviations } \\
\hline & \multicolumn{2}{|c|}{ Hierarchical Bayesian Method } & \multicolumn{2}{|c|}{ Interval Mapping Method } \\
\hline & $\begin{array}{l}\sigma_{1}=2.0 \\
\sigma_{2}=4.5\end{array}$ & $\begin{array}{l}\sigma_{1}=4.2 \\
\sigma_{2}=9.1 \\
\end{array}$ & $\begin{array}{l}\sigma_{1}=2.0 \\
\sigma_{2}=4.5\end{array}$ & $\begin{array}{l}\sigma_{1}=4.2 \\
\sigma_{2}=9.1 \\
\end{array}$ \\
\hline 2 & $\begin{array}{l}\text { Chrom } 3 \\
\text { M } 18(1.000)^{1} \\
\text { M } 19(0.987)^{1}\end{array}$ & $\begin{array}{l}\text { Chrom 5 } \\
\text { M } 35(0.586)^{1} \\
\text { M } 36(1.000)^{1}\end{array}$ & $\begin{array}{l}\text { Chrom } 3 \\
\text { M } 19(52.49)^{2}\end{array}$ & $\begin{array}{l}\text { Chrom } 5 \\
\text { M } 37(17.53)^{2}\end{array}$ \\
\hline 12 & $\begin{array}{l}\text { Chrom } 2 \\
\text { M } 15(1.000)^{1}\end{array}$ & $\begin{array}{l}\text { Chrom } 2 \\
\text { M } 11(0.989)^{1} \\
\text { M } 12(1.000)^{1}\end{array}$ & $\begin{array}{l}\text { Chrom 3 } \\
\text { M } 16(117.84)^{2} \\
\text { Loc } 2.5 \mathrm{cM}(63.05)^{2}\end{array}$ & $\begin{array}{l}\text { Chrom } 2 \\
\text { M } 13(106.39)^{2}\end{array}$ \\
\hline
\end{tabular}

Table 4. Two QTL Summary Table. $\left({ }^{1}\right.$ Final conditional activation probability. ${ }^{2}$ LOD score.)

\begin{tabular}{|c|c|c|c|c|}
\hline \multirow{3}{*}{ Effects } & \multicolumn{4}{|c|}{ Standard Deviations } \\
\hline & \multicolumn{2}{|c|}{ Hierarchical Bayesian Method } & \multicolumn{2}{|c|}{ Interval Mapping Method } \\
\hline & $\begin{array}{l}\sigma_{1}=1.5 \\
\sigma_{2}=2.5\end{array}$ & $\begin{array}{l}\sigma_{1}=2.0 \\
\sigma_{2}=4.5\end{array}$ & $\begin{array}{l}\sigma_{1}=1.5 \\
\sigma_{2}=2.5\end{array}$ & $\begin{array}{l}\sigma_{1}=2.0 \\
\sigma_{2}=4.5\end{array}$ \\
\hline 1,2 & $\begin{array}{l}\text { Chrom } 1 \\
\text { M } 5(1.000)^{1} \\
\text { Chrom } 4 \\
\text { M } 24(1.000)^{1}\end{array}$ & $\begin{array}{l}\text { Chrome } 2 \\
\text { M } 11(0.997)^{1} \\
\text { Chrom } 5 \\
\text { M } 36(1.000)^{1}\end{array}$ & $\begin{array}{l}\text { Chrom 4 } \\
\text { Loc, } 17.5 \quad \text { cM } \\
(21.73)^{2}\end{array}$ & LOD all $<11$ \\
\hline 1,2 & $\begin{array}{l}\text { Chrom 2 } \\
\text { M14 }(0.999)^{1} \\
\text { Chrom } 3 \\
\text { M } 21(1.000)^{1} \\
\text { M } 22(0.543)^{1}\end{array}$ & $\begin{array}{l}\text { Chrom 1 } \\
\text { M } 8(0.963)^{1} \\
\text { Chrom } 3 \\
\text { M18 }(1.000)^{1} \\
\text { M } 19(0.535)^{1}\end{array}$ & $\begin{array}{l}\text { Chrom } 3 \\
\text { Loc, } 65 \mathrm{cM}(33.94)^{2}\end{array}$ & $\begin{array}{l}\text { Chrom 3 } \\
\text { M } 19(16.37)^{2}\end{array}$ \\
\hline 2,12 & $\begin{array}{l}\text { Chrom 1 } \\
\text { M5 }(1.000)^{1} \\
\text { Chrom } 4 \\
\text { M } 24(1.000)^{1}\end{array}$ & $\begin{array}{l}\text { Chrom } 2 \\
\text { M11 }(1.000)^{1} \\
\text { Chrom } 5 \\
\text { M } 36(1.000)^{1}\end{array}$ & $\begin{array}{l}\text { Chrom } 4 \\
\text { M25 }(88.08)^{2}\end{array}$ & $\begin{array}{l}\text { Chrom 5 } \\
\text { M37 (93.55) }\end{array}$ \\
\hline
\end{tabular}

\section{Conclusions}

Many novel approaches have been developed for QTL analysis over the past 25 years. However, most methods assume only one observation per genotype, or equal variances within each genotype. Plant biologists have the luxury of cloning plants and creating replicates within each line. These replicates provide information about the QTL, but also on the variances within each line. Summarizing the replicates into one observation to utilize available software disregards the abundant information provided by the replicates. In these simple simulations of allowing the variances to have two values, we illustrate the importance of incorporating the different variance structures into the analysis. The additional complexity introduced by different variances within lines is easy to incorporate by using a Bayesian hierarchical model and is more 
appropriate in situations such as this. More extensive simulations to determine power and Type I error are needed, but these initial results are promising.

\section{References}

1. Sax, K.: The association of size differences with seed-coat pattern and pigmentation in Phaseolus vulgaris. Genetics 8, 552-560 (1923)

2. Lander, E.S., Botstein, D.: Mapping mendelian factors underlying traits using RFLP linkage maps. Genetics 121, 185-199 (1989)

3. Jansen, R.C.: A General Mixture Model for Mapping Quatitative Loci by Using Molecular Markers. Theoretical and Applied Genetics 85, 252-260 (1992)

4. Zeng, Z.B.: Theoretical basis for separation of multiple linked gene effects in mapping quantitative trait loci. Proceedings of the National Academy of Science USA 90, 10972$10976(1993)$

5. Wright, A.J., Mowers, R.P.: Multiple regression for molecular-marker: quantitative trait data from large F2 populations. Theoretical and Applied Genetics 89, 305-312 (1994)

6. Kearsey, M.J., Hyne, V.: QTL Analysis, A simple marker regression approach. Theoretical and Applied Genetic 89, 698-702 (1994)

7. Wu, W.R., Li, W.M.: A New Approach for Mapping Quantitative Trait Loci Using Complete Genetic Marker Linkage Maps. Theoretical and Applied Genetics 89, 535-539 (1994)

8. Sen, S., Churchill, G.A.: A statistical framework for quantitative trait mapping. Genetics 159, 371-387 (2001)

9. Lamon, E.C., Clyde, M.A.: Accounting for Model Uncertainty in Prediction of Cholophyll A in Lake Okeechobee. Journal of Agricultural Biological and Environmental Statistics 5, 297-322 (2000)

10. Zeng, Z.B.: Precision mapping of quantitative trait loci. Genetics 136, 1457-1468 (1994)

11. Zeng, Z.B., Kao, C.H., Basten, C.J.: Estimating the genetic architecture of quantitative traits. Genetic Research 74, 279-289 (1999)

12. Bao, H.: Bayesian Hierarchical Regression Model to Detect Quantitative Trait Loci. UNCW Thesis (2006)

13. Satagopan, J.M., Yandell, B.S., Newton, M.A., Osborn, T.C.: A Bayesian approach to detect quantitative trait loci using Markov chain Monte Carlo. Genetics 144, 805-816 (1996)

14. Sillanpaa, M.J., Arjas, E.: Bayesian mapping of multiple quantitative trait loci from incomplete inbred line cross data. Genetics 148, 1373-1388 (1998)

15. Sillanpaa, M.J., Corander, J.: Model choice in gene mapping, what and why. Trends in Genetics 18, 301-307 (2002)

16. Xu, S.: Estimating Polygenic Effects Using Markers of the Entire Genome. Genetics 163, 789-801 (2003)

17. Yi, N., Xu, S., Allison, D.B.: Bayesian model choice and search strategies for mapping interacting quantitative trait Loci. Genetics 165, 867-883 (2003)

18. Boone, E.L., Ye, K., Smith, E.P.: Evaluating the Relationship Between Ecological and Habitat Conditions Using Hierarchical Models. Journal of Agriculture, Biological, and Environmental Statistics 10(2), 1-17 (2005)

19. Bjornstad, A., Westad, F., Martens, H.: Analysis of genetic marker-phenotype relationships by jack-knifed partial least squares regression (PLSR). Hereditas 141, 149 165 (2004) 
20. Broman, K.W., Wu, H., Sen, Ś., Churchill, G.A.: R/qtl, QTL mapping in experimental crosses. Bioinformatics 19, 889-890 (2003)

21. Simmons, S.J., Piegorsch, W.W., Nitcheva, D., Zeiger, E.: Combining environmental information via hierarchical modeling, an example using mutagenic potencies. Environmentrics 14, 159-168 (2003)

22. Boone, E., Ye, K., Smith, E.P.: Assessment of Two Approximation Methods for Computing Posterior Model Probabilities. Computational Statistics and Data Analysis 48, 221-234 (2005)

23. Loudet, O., Chaillou, S., Camilleri, C., Bouchez, D., Daniel-Vedele, F.: Bay-0 x Shahdara recombinant inbred lines population, a powerful tool for the genetic dissection of complex traits in Arabidopsis. Theoretical and Applied Genetics 104(6-7), 1173-1184 (2002)

24. Lynch, M., Walsh, B.: Genetics and Analysis of Quantitative Traits. Sinauer Associates, Inc., Sunderland, MA (1998) 